\title{
Emergence and Playfulness in Social Games
}

\author{
Ben Kirman \\ Lincoln Social Computing Research Centre \\ University of Lincoln, LN6 7TS, UK \\ +44 (0)1522837083 \\ bkirman@lincoln.ac.uk
}

\begin{abstract}
Social Games, built and played on social networks such as Facebook, have rapidly become a major force in the world of game development, and the top social games today claim more players than any other online game on any format.

As social games begin to mature from their roots as simple playful social toys and into the products of big business, the patterns and mechanics used in the design have begun to be formalised.

In this paper, it is argued that experimentation and playfulness is still a very important part of the play experience and a valuable source of fun. As game designs explore the space opened by the new genre of social games, it is vital for designers to leave "gaps" in the design to allow for playful and serendipitous experiences to emerge from the activities of the players.

To support this argument, Caillois' classification of play is used as a lens through which social games can be examined. Examples of paidic, playful and emergent play are presented from popular social and offline games, and a detailed case study of paidic play in a new social game is presented from the designer's perspective. Interviews from participants to an open trial are discussed, and their experiences in creating their own playful experiences and goals within the formal structure of the social game design are explored.
\end{abstract}

\section{Keywords}

Social Games, Game Design, Paidia, Playfulness, Ludology

\section{INTRODUCTION}

Social Games, popularly defined as games played on and around online social networks, have had a meteoric rise in popularity since the genre was effectively created in 2007.

The most popular, Farmville had just over 77 million unique players in April 2010 [7] and other top social games count their players in the tens of millions. The stereotypical views of game players as being mostly adolescent males interested only in shooting games has become profoundly challenged. According to recent research [6], the average FarmVille player is 31 (33\% over $35)$ and equally likely to be female as male $(51 \% / 49 \%$ respectively).

As the audience for social games grows, the need to purposefully design game experiences that appeal to this particular type of gamer has become important. The huge success of the top few games have led to a succession of copycat games trying similar ideas with new mechanical tweaks. The academic field of Games Studies is still trying to catch up and understand what these social games are and how gamers behave differently while playing (e.g., [11]).
Over their short evolution, Social games as a sub-genre of online games have become rather more formalised. In their origin they were built around the social network, as a purely playful tool to enhance your social experience with friends on the social network [14]. However, now the most popular applications are now fullfledged game "experiences" with vast amounts of content and hours of playtime that perhaps have more in common with traditional computer games than their social forebears - for some players the current games may have more value than the social network itself.

Rather than being developed by small teams of "bedroom" programmers, now social games are big business, with major players such as Zynga and EA/Playfish [12].

The design of games could be in danger of becoming homogenized as designers try to duplicate the success of the first "huge" social games like Mafia Wars and Farmville by using similar mechanics on a smaller budget. The game experiences become simplified in order to appeal to the new demographics, that may not have been exposed to a lot of games before, and as such may not be "game literate" or be able to immediately recognise traditional patterns and structures used in games design for other platforms.

However, despite the new opportunities and challenges created by the developing genre of social games, some important ideas about game design theory, that were first proposed before the invention of Pong, remain just as relevant today.

\section{THE IMPORTANCE OF PAIDIA (or PLAYFULNESS)}

In the seminal work Man, Play and Games [5], the sociologist Roger Caillois described all forms of play, including games, as being positioned on an axis between two extreme points: Paidia and Ludus. He described the concept of Paidia as "a primary power of improvisation and joy" compared to its polar opposite, Ludus, for play heavily defined by strict rules, which is defined by "a taste for gratuitous difficulty". Every game conceptually sits at some point on the scale, depending on how much of the experience is driven by a formal system of rules.

Man, Play and Games was written long before digital games became popular, but still applies just as well in both the analogue and digital worlds. When thinking about playfulness in all kinds of games, it is very helpful in thinking about the types of play encouraged and enforced by the game design. Caillois' classifications of play simply serve as a lens through which we can analyse play.

Using examples from "traditional" console games to illustrate these concepts, The Guitar Hero and Rock Band series' of games are examples of play that is further towards the ludic end of Caillois' conceptual scale. In these games, you either succeed in 
following the instructions of the game as best you can, or you fail. There is no room for individual interpretation or play within the rules as imposed by the games. The rules can't be bent and there are little to no meaningful choices offered to the players. In the words of Gonzalo Frasca, "Clearly defined goals do not generally leave much room neither for doubts nor for contesting that particular objective"[9] This does not mean the games aren't fun, which they evidently are, but that the source of that fun is not the same as it is in other games that rely less on Ludus.

In non-electronic games, such as those found on every decent schoolyard, play that is at the Paidic end of the scale are supported natively because the rules are highly flexible and defined largely socially. However, when games are mediated by computer systems, the inflexible way the systems are built means there are few such "holes" that allow rules to be bent and adjusted on-thefly.

At this end of the scale, Grand Theft Auto, The Sims and other "sandbox" games can be found since they are designed to be more freeform play experiences, where players are allowed, or even encouraged, to explore the environment and find their own source of amusement.

In the words of Caillois,

"[Paidia] covers spontaneous manifestations of the play instinct: a cat in a ball of wool, a dog sniffing, and an infant laughing at rattle represent the first identifiably examples of this type of activity"

The noted game designer Chris Bateman interprets Paidia as the "anarchic nebula from which all play originates" [3] and argues that the first moments of playing any new game are highly playful, until the user learns the ludic rules of the game and falls into the structured patterns of play [2].

However, it is important to note that Paidia is not just found in games that are designed specifically to allow for this form of play. Just about every game supports paidic play in some form (even if it is just using the game disc as a Frisbee!), but through design choices, some games support this style of play more explicitly.

\subsection{The Freedom to Fail}

A lot of games give the illusion of freedom, but the formal structure of "how the game should be played" only allows freedom within these carefully constrained limits.

True freedom in design gives the players the opportunity to wilfully fail at the task they have been given. By giving the player freedom to do this, they can generate their own goals that are not dependent on playing within the constraints of the "normal" way a game is played.

Salen and Zimmerman call this freedom for explorative play a form of "emergence" in a design [15], something that must be allowed for within the formal game design and possibly enabled by tools as channels for sharing newly discovered opportunities for play between players.

"One of the sweetest pleasures as a game designer is seeing your game played in ways that you did not anticipate" (p540)

As a contrary example, in Rock Band, a group of players may decide they wish to play their own version of a song, only including the lead guitar at some sections, and giving more room for extended drum solos where they don't exist in the original song. If they tried this, when the game detects the lead guitarist is not playing notes where they should, it would interpret the playfulness as "failure" and cut the game short. This would then deprive the players the chance to enjoy their self-directed paidic experience.

Grand Theft Auto (GTA) is an example of a large commercial game that gives players the freedom to fail and therefore far more freedom over their experience of the game. In the original game released in 1997, the players only had a limited number of lives, and when the player died too often, the game would end in failure. In the sequels, the game's designers have learnt that the player has the "right" to die, and to die as often as they please (e.g. by repeatedly throwing themselves off the top of the tallest buildings in the game-world just because it sounds funny when the avatar hits the pavement). This gives players the freedom to create their own fun experiences within the game, without fear of intervention by the game design. The Grand Theft Auto series is not without criticism on this point, however. For example, in all of the iterations of the game, players are punished for not following story points by being denied access to the complete game world. Large swathes of game world ripe for exploratory, paidic and emergent play are hidden until the player operates the game "correctly" by playing through a series of linear and controlled missions as part of the story arc.

These examples of paidia and ludus are taken from popular console games, however it is very important to stress that paidia and playfulness are present in all kinds of games, regardless of platform.

\subsection{Freedom is not Necessarily Design Complexity}

When talking about game design using terms like "player freedom", the implication is that the game design must therefore become much more complicated in order to allow paidic or playful behaviour. As Bateman says, the approach taken by the GTA developers to add more and more opportunities for paidia in their complex environments can be "devastatingly expensive" [3]. However (as Bateman acknowledges), this is not always the case. Although it can be argued that the type of player who enjoys paidic experiences would also enjoy exploring and messing around in a large, open and highly detailed virtual world (that has cost many thousands of man-hours to develop), it is short-sighted to view that as a requirement for that kind of playful fun to occur.

In actual fact, all that is required is a lack of barriers. It is still probably a good and solid design idea to guide new players along the conceptual footpath or "vector" of normal play, to make sure they understand the designed goals of the game and how to achieve formal rewards. However, the players should be allowed to stray from the path if they wish, and do so without fear of punishment by the game (either formally or socially).

It is these "design gaps" that give players the ability to create fun, especially in social and multiplayer games, rather than having the "fun" predetermined, regardless of whether the game is a simple playground game, a multi-million dollar console title or even a simple web-based social game.

\subsection{Freedom does not Necessarily Make the Game Easy}

Another assumption that can be made, is that paidic play is easy, cheating, or somehow a "way out" of the perceived challenge of a game. While this is strictly true, it should be regarded as a positive effect - the freedom of paidic play can also quickly lead players to 

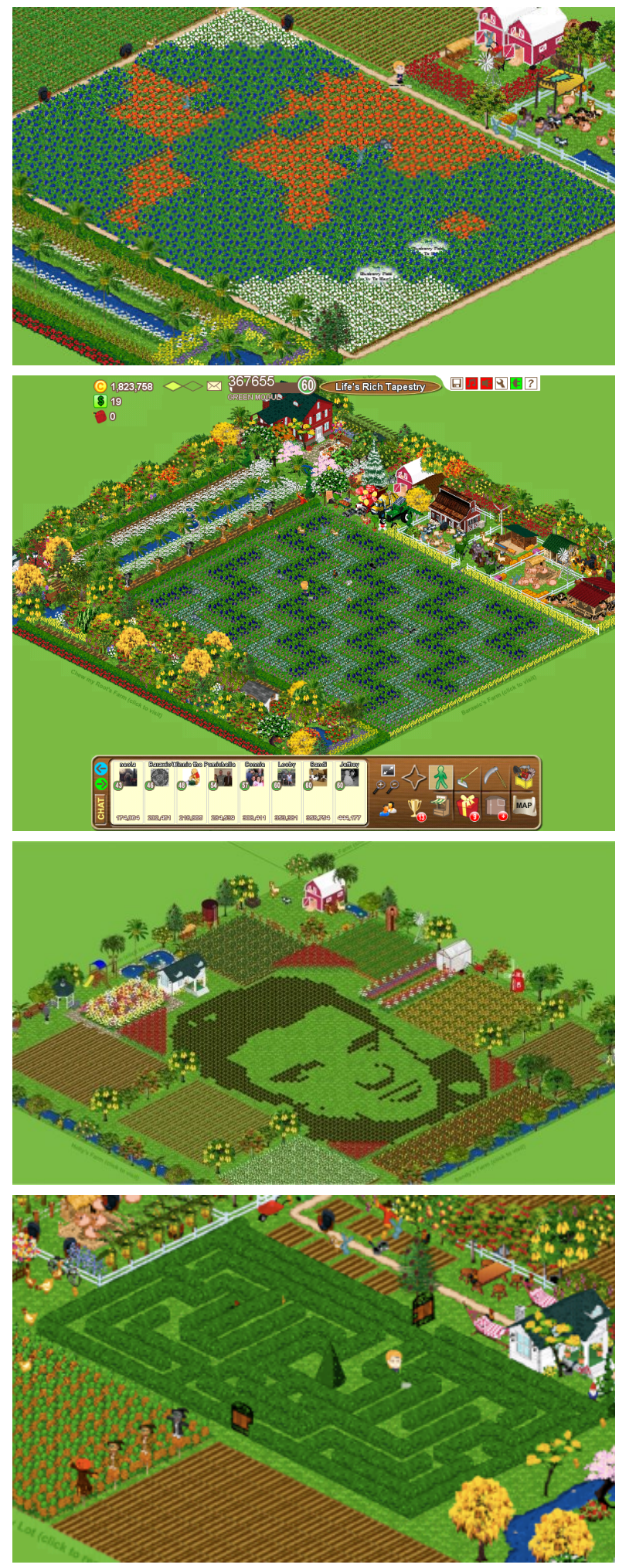

Figure 1 - Creative Agriculture in Farm Town

develop new systems of rules in order to create challenges even greater than those posed by the formal system of the game itself.
For example, consider the emergence of "speedruns" [16]. In this activity, a player chooses to try and complete a game, or section of a game, as quickly and flawlessly as possible. Although this form of play is not implied by the game rules, it has emerged as a way to demonstrate skill far above and beyond what is required to win the game "formally" within the usual parameters since it requires superb timing and memorisation skills. Since emerging as a paidic activity played around game rules, the speedrun community has since developed its own formal rule system to allow competition, itself becoming a more ludic activity as time progressed.

Other examples of players subverting the rules of games to make things purposefully more difficult can be found for many games. In the famously difficult Nethack, a fiendishly hard dungeon crawling game, some players make the game even more difficult by choosing to play as strictly vegetarian, vegan or even pacifist characters [13].

\section{PLAYFULNESS IN SOCIAL GAMES}

As far as game design complexity goes, it is fair to consider that the new breed of social games lie on the simpler side. Since they are nearly always web-based, the graphics and interactions are limited to what is workable on the typical web browser. As the demographics of the average player shifts away from those who may have high literacy of games, game mechanics and their own high-powered dedicated gaming machines; and toward people who, for example, may play on restricted or underpowered machines used for playing during lunch hour in the office; there are limits to complexity of designs (both graphically and conceptually) that can become popular in this context.

Despite these limitations, some games have been designed, either purposefully or by accident, with low barriers to non-linear play. Giving the players tools to create their own experiences within the (even limited) game world has allowed players to engage in their own paidic and playful experiences.

\subsection{Creative Agriculture in Farm Town}

Farm Town [8] is one of a range of popular farming themed games found on Facebook. In these games, players own a farm, and must maintain both the business and the finer details of arable and pastoral farming. This includes buying land and animal feed, planting crops and harvesting them for profit when the time is right. Farm Town has nearly 9 million unique players each month [7]. One of the key mechanics of Farm Town is that each user has a particular amount of virtual land on which they can build their farm, and the player has complete control over where fields, buildings and pastures are placed within this virtual space. The location of crops has no direct impact on the player's ability to be a successful farmer (e.g. crops are no less likely to produce a harvest if very far away from the farm buildings) so this control has no direct in-game impact.

The logical and mathematically rational strategy would be to place the elements of the farm in an arbitrary fashion based on the order of construction, and the developers would have been forgiven if this had been an automatic feature, however, some players have taken this "gap" as an opportunity to show their creativity while playing the game.

Figure 1 shows several examples of how players have contrived to arrange their farms specifically for aesthetic or artistic purposes. The examples show a map of the world, a reconstruction of tile patterns taken from the mosaics at the Alhambra in Granada, the 


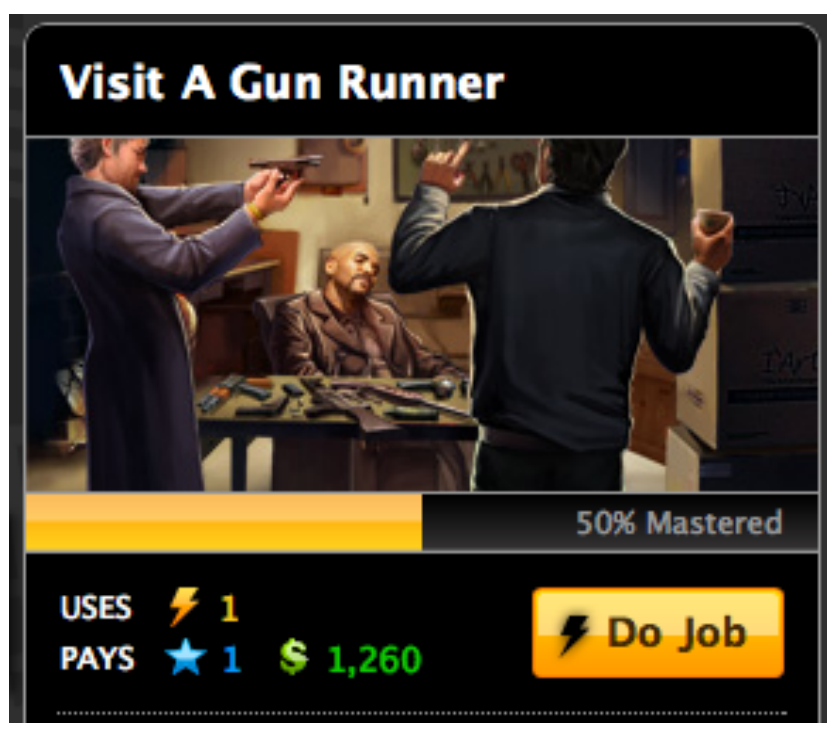

Your mission: Plunder Enchanted Motorcycle Hunter Stranglers Wielding Invisible Bandyclefs

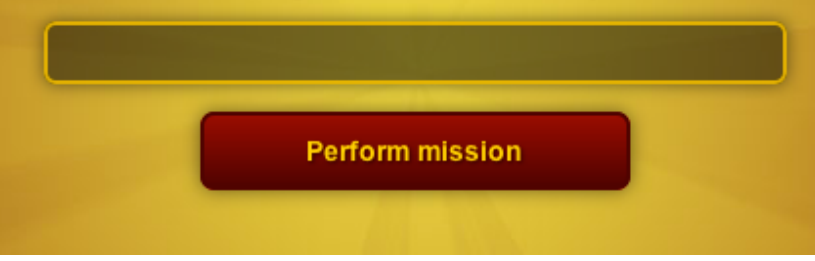

Figure 3 - Mafia Wars and parody Progress Quest; Simple interactions with rich game narratives

likeness of Elvis and finally a maze based on the $18^{\text {th }}$ century garden mazes found at palaces and stately homes such as Tatton Park and Hampton Court in England. Players have formed groups within Facebook in order to share hundreds of their creations with one another, sharing tips and techniques for how to use the tools of the game to create various effects ${ }^{1}$. The maze is of particular interest, the creator reports:

"[the maze] actually works - the avatar makes its way to the centre of the maze if you click by the cone hedge "

The maze creator has taken advantage of the fact that the software implements a pathfinding algorithm for avatars within the game, to allow them to automatically walk between any two locations on a farm based on mouse clicks by the user. Although they may not be aware of the technical details of the pathfinding algorithm, they have devised mazes as a way of "testing" its efficiency at finding the shortest paths. Therefore the original design choice made by the developers to use a pathfinding algorithm to determine avatar movement (instead of, say, just direct control using the cursor keys) has led to another serendipitous form of fun that has emerged through paidic play by some of the users.

\footnotetext{
${ }^{1}$ E.g. http://www.facebook.com/\#!/group.php?gid=91934065717
}

Farm Town is just one example of games that allow the possibility to create such "pixel art" from the building blocks provided in the game. The key point for these games that allow this particular form of play is the provision of virtual space that allows players control over individual graphical elements. Farms, villages and night clubs (as seen in other games) could have easily been represented by a simple list of buildings and items, but the simple virtual grid allows for exciting creativity and play generated by the players themselves. This is an example of a "design gap" where the players have enough freedom to express their own playful nature despite there being no direct benefit to them in the formal game structure, such as gaining points or rewards.

\subsection{Restrictive Social Games}

Farm Town, FarmVille, and other games that have these "design gaps" are popular, but there unfortunately the majority of social games appear to be highly restrictive. In other words, the high barriers that guide players along their game experience can be almost impossible to overcome. Players are forced to play along with the strict narrative path of the game and are unable to create their own playful experiences for themselves.

Games like Mafia Wars, Vampires, West Wars and many others are driven by one major form of interaction - semi-random "missions" that must be completed by repeatedly clicking the same button again and again. The descriptions and rich game narrative of the missions change over time but the actual player activity remains the same - clicking a button. There is no capability for players to escape out of this design loop, and therefore no "design gaps" that allow for playfulness as paidia to appear.

Although these games have been criticized and parodied (e.g. Figure 3) for their simple and repetitive nature, it is very important to note that these games are still fun for millions of users. The narrative is strong enough that it does not matter to the players that there is low depth of interactivity. This form of structured play and enjoyment is perfectly valid (and should not be treated dismissively), however in Caillois' classifications these games sit very far on the side of ludus and are presented as contrast to other games presented here since they offer little to no options for paidic play.

\section{EMERGENCE BY ACCIDENT: PASION FRUIT}

Emergence and playfulness in social games clearly happens, but it is unclear about the role of the game designer in creating or enabling these behaviours. In order to explore this, we present an example of paidia and playfulness that emerged in one of the authors' games. In particular how unintentional "design gaps" led to emergence, and how this was observed in real time across a complete player-base.

As part of our own research into mediated social communication [4], a social game was developed to study interactions in games deployed on social networks such as Facebook. It was deployed online (at www.pasionfruit.eu) and interactions recorded during an 11-week formal public trial. The research questions that PASION Fruit was designed to answer were based around group cooperation within online games.

The core theme of PASION Fruit is environmental sustainability. Each player, upon registering to play the game, is asked to choose a real location for his or her garden. This is provided either by address/postal code or by clicking a location on an interactive 


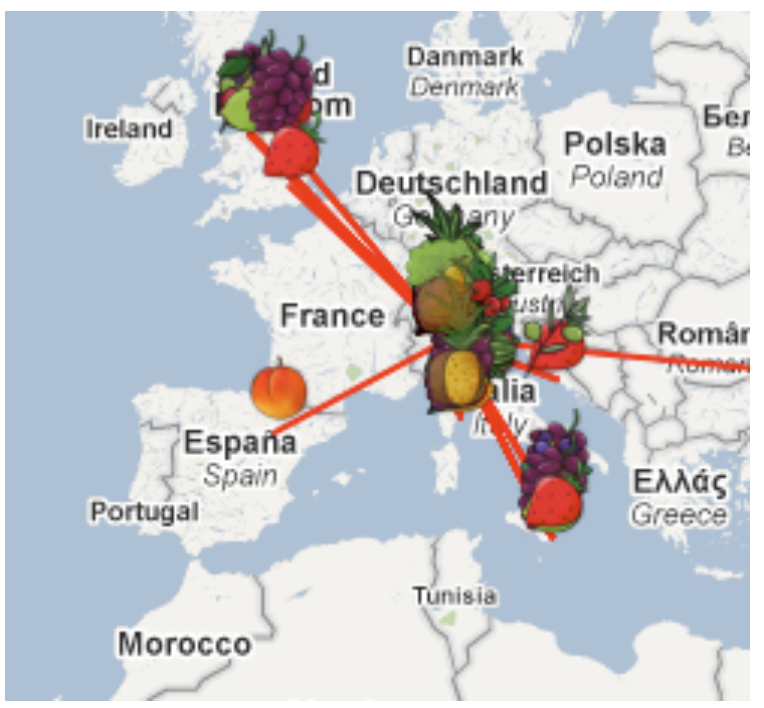

Figure 6 - Players can see histories of distances fruit has travelled and $\mathrm{CO} 2$ generated

map. PASION Fruit is hosted on its own private webserver, however through the service "Facebook Connect" it is able to connect to players' social networks on Facebook.

Initially, each garden contains five fruit trees of the same type, depending on the country in which the garden is found. For example, gardens planted in the UK will grow one of Apples, Pears or Strawberries natively. Gardens in Italy will grow Lemons, Grapes or Olives. There are twenty different types of fruit represented in the game, and the relationship between fruit types and countries were defined manually by the designer.

The formal goal of the game is to gain points based on the diversity of fruit types within your garden, however this is balanced against the environmental cost of transporting fruits long distances. Since each country only grows a certain subset of fruits natively, in order to be successful players are forced to transport more exotic fruit from distant locations and pay an environmental cost represented by $\mathrm{CO} 2$ expenditure.

A main feature of the design is that players don't get to choose what fruit they plant in their garden - they can only receive gifts of fruit trees from other players. They can request types of fruit using the comments/messaging system within the game, but whether the other player will send the desired fruit or not is unknown. Secondly, the player sending the gift bears the entire cost of $\mathrm{CO} 2$ emissions generated by sending the fruit the distance between the two gardens. Therefore, when sending a gift of one of your valuable fruit trees to another player, you not only lose the tree itself, but you lose the points it contributed towards your own garden's diversity, you pay the environmental cost (and therefore cost in score) to send it however far it must travel, and get no direct benefit from your act.

PASION Fruit provides a wealth of social history tools for players to be able to see the past behaviour of other players, to help them make informed decisions about who to send gifts to - do they need this kind of fruit? Are they likely to return a gift? Do they have a type of fruit I need? Will they be willing to lose it?
An abstract score is generated in real-time for each player based on the diversity of their garden, and the amount of CO2 emissions they have generated as a result of gifts they have sent. This score is then ranked against others in a high score table so players can see how they are performing.

\subsection{Public Trial}

PASION Fruit was opened to the public in a trial lasting 11 weeks, starting in mid-February 2010. Initially, a handful of participants were recruited in Italy and the UK and asked to play the game. Since registration was publicly open, and the game integrated with the social network Facebook, the player-base was permitted to grow as a natural viral or snowballing effect as would be experienced by a typical social game on the site. By the end of the trial, there were 99 active users who had between them generated 3922 gifts of fruit between one another.

Although the trial had gathered the data necessary for the separate research on the social behaviour of game players, unusual behaviour had also been noticed in the game that didn't fit the profile of what was expected.

The introduction to the game had a clear tutorial, and the game mechanics were fairly straightforward, so some behaviours within the game were surprising. In particular some players had outrageously large $\mathrm{CO} 2$ expenditure, and therefore very low (even negative) scores. In order to learn more, a qualitative questionnaire was sent to all participants asking for them to explain how they had played the game. 30 responses were received from players.

\subsection{Self-Directed Goals and Playfulness in PASION Fruit}

As stated earlier, PASION Fruit was designed specifically to answer research questions about group cooperation in online games. However, while running the trial it quickly became clear that the players were not simply playing the game as designed, but breaking out of our carefully designed experience to create their own fun.

According to the responses to the questionnaire, there appears for a subset of PASION Fruit players to be an alternative goal, not related to score.

Since the game had 20 different types of fruit, and the players had space for 20 trees in their gardens, some players created an informal challenge to have all 20 types of fruit in their garden at any one time. This was no small feat, since every day each fruit tree has a chance of dying, and a small chance of breeding a new tree. Therefore, with no space for new trees to breed, and existing and perhaps rare fruit trees dying, maintaining a garden full of individual trees is very difficult. Maintaining a garden with all 20 fruit trees at once is even harder, and only sustainable for a few hours (before another tree dies).

$\mathrm{CO} 2$ expenditure has a huge impact on score in PASION Fruit, so for a player trying to be competitive, it is not in their advantage to collect a huge variety of fruits. The recommended strategy was one of sustainability, choosing to maintain local sources of rare fruits with players in the local regions. As described by a respondent:

"I tried to keep a mix of fruits locally. I worked with people locally to keep the rare fruits alive. After a while i would get rid of any fruits that were prolific in the area by deleting them rather than sending them on to people who probably didn't 

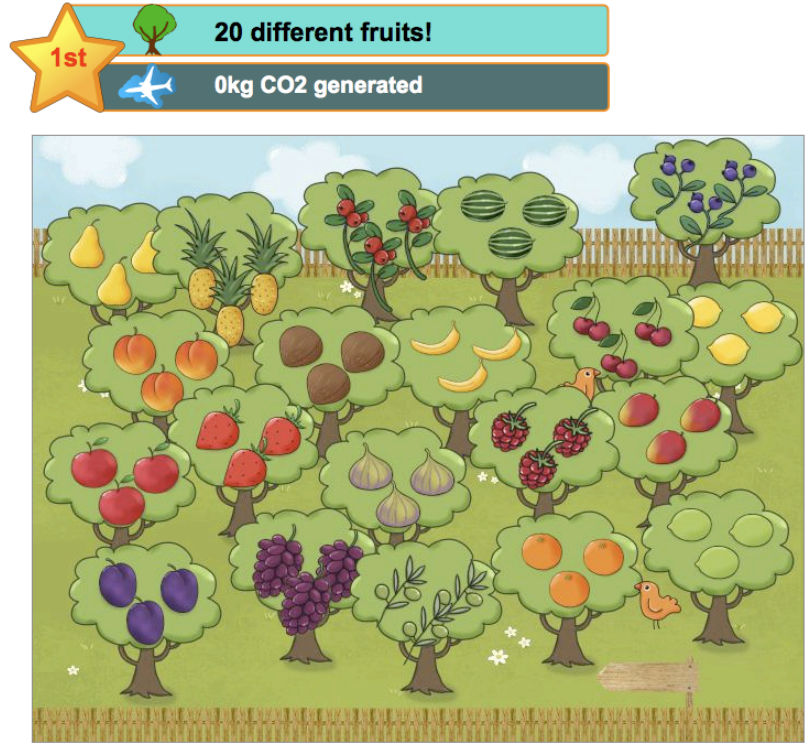

Figure 8 - The "Perfect" PASION Fruit Garden

want them. At first i sent these locally prolific fruits to other countries in hope to get some different fruits in return. Once all the fruits were in the country $i$ stopped sending abroad in hope to keep my carbon count down." (P5)

The players choosing to challenge themselves to get all 20 fruit were doing so in the knowledge that it would irrecoverably damage their score due to the amount of $\mathrm{CO} 2$ emissions generated. This was directly against the designed goals of the game.

One player reported they had set a goal that took this idea to the extreme:

"Be \#1 with the highest possible score. I.e. No CO2." (P6)

This player had realised that getting 20 distinct trees would be difficult without generating huge amounts of $\mathrm{CO} 2$, had set a new
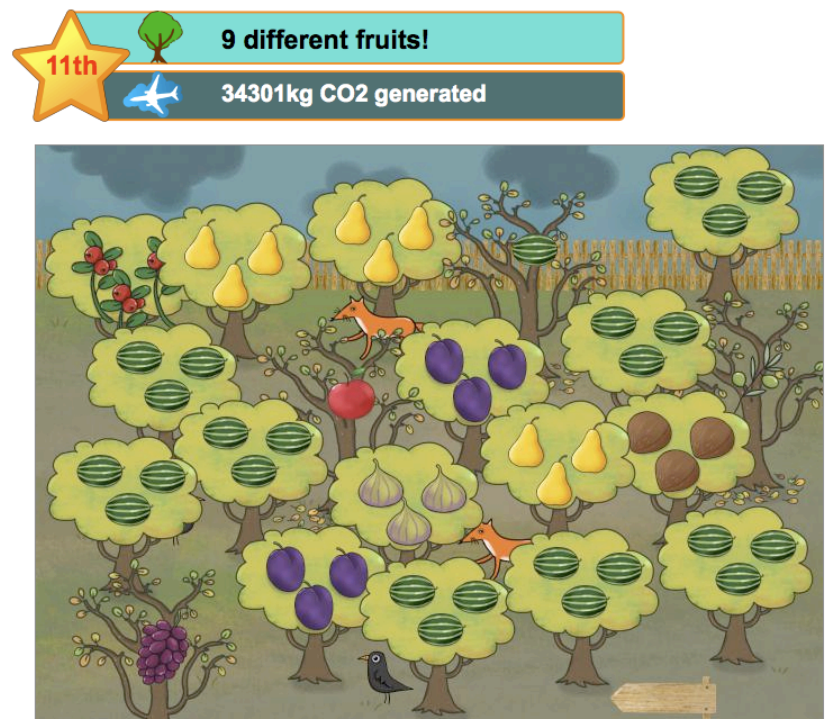

Figure 9 - Diversity, but at huge environmental cost goal: achieve the maximum possible score.

Since the game mechanics of PASION Fruit only define that the sender of a gift has to pay costs in terms of $\mathrm{CO} 2$, it is technically possible to have a garden without any $\mathrm{CO} 2$, by never sending a gift. This player went on a mission to ask other players within the game to sacrifice their own score in order to help him achieve this impossible task. Surprisingly, enough of the other players wanted to see him succeed, that quickly he managed to achieve, not only 20 different fruits, but simultaneously with a $\mathrm{CO} 2$ expenditure of 0 . This gave him the maximum possible score of 200,000 points for a few short hours before the trees started to die and the score decreased.

\subsection{Emergence by Accident}

From the designer's perspective, players behaved erratically in PASION Fruit - in particular, the emergent ideas of players to ignore their scores and focus on self-directed goals for getting varieties and types of fruit in their garden was clearly a result of paidic experimentation within the game rules. These players wanted to explore the boundaries of what was permitted within the game and what could reasonably be fun to achieve. In doing so, this emergent challenge was created that a significant portion of players felt was, perhaps, more fun than following the formally defined game objectives.

In this respect it is pleasing the players have found new and novel ways to enjoy the game that were previously unexpected, but it challenges the game designer to think, "what could we have done to allow more of this behaviour?"

Despite not having the resources of a large game developer to make sweeping changes to the design, subtle changes could still encourage further playful and emergent activity within the confines of the game functionality.

\section{DESIGN GAPS}

The emergence of playful behaviours in social games appears to be based on what we term "design gaps" - that is, some possibilities for players to have an effect on the game world that may not have direct benefits or cost (and may have been unintentional on the part of the designer). For example, being able to manually place items in a virtual world, or being able to customize avatars, or even providing scope for player-defined challenges are all kinds of "gap" in the experience as defined by the formal, ludic, game structure.

It is also possible for some designs to be so tight that there is little or no scope for playfulness within the formal game structure. The examples of highly ludic "restrictive" social games such as Mafia Wars appear to lack any of these "gaps" for players to exploit.

Even in games that contain such "gaps", the possibilities become closed as barriers to use them increase. If a player is punished for exploring a game space, or going "off the beaten path", the motivation to do so may be reduced; the player is effectively discouraged from creating more playful experiences.

In the case of PASION Fruit, every action in the game has a direct consequence, and emergent play only occurred in spite of these design decisions. Had there been lower barriers on the "design gaps" - where lack of ludic barriers mean the game does not directly punish the player for failing to conform to the rules, experience suggests we might have seen a much more active and paidic in-game society. 


\section{DISCUSSION}

Social games are the latest in a long history of innovations in games design. In just a few short years, the genre has quickly become one of the most popular forms of gaming available. The audience demographics have shifted dramatically from what had become the norm in digital games, and along with it the expectations, skills, likes and dislikes of the players.

Despite this seismic shift in the landscape of game design theory and practice, core theories of games, such as those proposed by Caillois in the mid $20^{\text {th }}$ century, are still as relevant today as they have been throughout the evolution of games as a medium.

In this paper, one particular aspect of Caillois' theories of play, Paidia, has been reintroduced and used as a lens to examine the social games phenomenon. Specific examples have been taken from popular social games in order to illustrate how, even in simple social games, paidia and playfulness can still be an important part of the game experience. This is contrary to the idea that playfulness requires significant extra development in order to support (e.g. as sandbox-style console games do)

Another social game, PASION Fruit, has been presented as a case study with more examples of playfulness on the part of the players to create more challenging experiences that were not a part of the game design. This designers' eye view demonstrates that the design process can lack any intentional will to support playfulness in social games - paidic and playful experiences will be created by the players themselves using whatever tools are available. However, as in the case of PASION Fruit, too many design barriers can directly punish the players who try to exhibit their playful desires. As seen in even more restrictive social games such as Mafia Wars, even if the games are fun as is, if there is no player freedom in the design, it can be argued that playfulness and paidia are prevented from emerging.

Although social games as a genre offer great new challenges and opportunities for novel game design, it is still vitally important to remember that the players are also capable of creating their own sources of amusement. Paidia and playful explorative activities within the confines of a system of rules, is perfectly capable of leading players to create new emergent meta-games with their own rule-sets. It appears that given the chance, players will find ways to subvert the formal rules of a game by exploiting "design gaps" in order to find new and exciting ways to have fun. However, the capabilities of the players is still limited by the tools available - the fewer the number of barriers and restrictions on player behaviour, and the more actions a player is able to take in a game, the more enabled the players become to define their own fun as games-within-the-game beyond what the game designer planned.

\section{ACKNOWLEDGMENTS}

PASION Fruit was designed as part of the PASION Project which was funded under the Presence II Initiative in the Future
Emerging Technologies within the European Framework VI Programme.

\section{REFERENCES}

[1] AppData Facebook Application Metrics, www.appdata.com

[2] Bateman, C. and Boon, R. 2006. $21^{\text {st }}$ Century Game Design, Charles River Media

[3] Bateman, C. 2005. The Anarchy of Paidia, accessed 4/2010: http://onlyagame.typepad.com/only a game/2005/12/the an archy of $1 . h \mathrm{tml}$

[4] Brugnoli, M.C., Morabito, F., Walker, R. and Davide, F. 2006. The PASION Project: Psychologically Augmented Social Interaction over Networks, PsychNology 4:1

[5] Caillois, R. 1961. Man, Play and Games, The Free Press

[6] Caoili, E. 2010. Study: 21 Percent of Gamers Aware of FarmVille Play it Daily, Gama Sutre, online: http://www.gamasutra.com/view/news/28322/Study 21 Perc ent_Of_Gamers_Aware_Of_FarmVille_Play_It_Daily.php

[7] Developer Analytics, Farm Town Report, April 2010 http://www.developeranalytics.com/app.php?id=56748925791

[8] Farm Town, Accessed April 2010: www.facebook.com/apps/application.php?id=56748925791

[9] Frasca, G. 2003. Simulation versus Narrative. The Game Theory Reader, Routledge

[10] Järvinen, A. 2009. Game Design for Social Networks, Accessed april 2010: http://www.mygamestudies.com/content/game-design-socialnetworks-part-1

[11] Kirman, B and Lawson, S. 2009. Hardcore Classification: identifying play styles in social games using network analysis. Lecture notes in Computer Science 5709, Springer

[12] Miller, C C. and Stone, B. 2009. Electronic Arts Buys Playfish, a Social Games Start-Up. New York Times November $9^{\text {th }} 2009$, New York

[13] Nethack Voluntary Challenges, Accessed April 2010: http://www.steelypips.org/nethack/conduct.html

[14] Rao, V. 2008. Facebook Applications and playful mood: the construction of Facebook as a "third place". MindTrek 2008, Tampere, Finland

[15] Salen, K. and Zimmerman, E. 2004. Rules of Play: Game Design Fundamentals, MIT Press

[16] Speed Demos Archive, Community Rules, Accessed April 2010: http://speeddemosarchive.com/lang/rules_en.html 